\title{
Correlation of Aluminum Hole Centers with Hydrogen Radicals from $\gamma$-Irradiated Quartz of Different Origins
}

\author{
Tetsuo Hashimoto, ${ }^{*, a}$ Hirotomo Hase, ${ }^{\mathrm{c}}$ Duk-Guen Hong, ${ }^{\mathrm{a}}$ Hiroki Fujita, ${ }^{\mathrm{b}}$ and Hajime Katayama ${ }^{\mathrm{b}}$ \\ ${ }^{a}$ Department of Chemistry, Faculty of Science, Niigata University, 8050 Ikarashi, Niigata 950-2181, Japan \\ ${ }^{\mathrm{b}}$ Graduate School of Science and Technology, Niigata University, 8050 Ikarashi, Niigata 950-2181, Japan \\ ${ }^{\mathrm{c}}$ Research Reactor Institute, Kyoto University, Kumatori, Osaka 590-0451, Japan
}

\section{Received: February 10, 2000; In Final Form: March 6, 2000}

\begin{abstract}
In all quartz samples having different origins, radiation-induced hydrogen radicals (atomic hydrogens) were observed in ESR measurement at $77 \mathrm{~K}$ in addition to intense Al-centers. An obvious positive correlation was recognized between the hydrogen radical intensities and the reduction of the Al-centers after warming up to room temperature, although there was no clear relationship between hydrogen signals and initial Al-centers at $77 \mathrm{~K}$. In isochronal annealing treatments the hydrogen radicals were gradually decreased with increments of temperature and completely disappeared after warming up to room temperature. Similar behavior was shown in the Al-centers, but a few percentages of which were still remained at room temperature. The Al-centers remained gave a linear relation to the blue thermoluminescence (BTL) intensity. We concluded that the hydrogen radicals arising from radiolytic products of $\mathrm{OH}$ and molecular water impurities could operate as a "killer" of radiation-induced Alcenters related intimately to emission of BTL in natural quartz.
\end{abstract}

\section{Introduction}

Blue-thermoluminescence (BTL) around 470nm wavelength from natural quartz minerals exposed to ionizing radiation has been attributed to the recombination of Al-hole centers $\left[\mathrm{AlO}_{4} \mathrm{~h}^{+}\right]^{0}$ with electrons released from trapped sites. ${ }^{1}$ However, negative or meaningless correlation between $\mathrm{Al}$ impurity contents and BTL intensities has been often met from a variety of natural quartz of different origins and even from different segments in a single rock crystal. ${ }^{2}$ In the recent papers, ${ }^{3,4}$ the aluminum distributions on the quartz slices, which were visualized by using an EPMA (electron probe micro-analyzer), have been found to offer somewhat negative correlation with both the BTL-stripe and the radiation-induced color center image (CCI) patterns. Some segments from the slice showed the obvious negative-correlation between the radiation-induced phenomena and both $\mathrm{Al}$ and $\mathrm{OH}$ (probably due to dangling bonds and molecular water) impurity concentrations, whereas Al-hole centers in the ESR-spectra gave a positive correlation with radiation-induced phenomena. ${ }^{5}$

Additionally, the formation of Al-hole centers has been also confirmed to be seldom in Brazilian hydrothermal quartz with a high content of $\mathrm{OH}$ and molecular water in the infrared absorption spectra. ${ }^{6,7}$ The similar role of $\mathrm{OH}$ and molecular water impurities on radiation-induced phenomena was confirmed on a slice specimen from the Japanese twin quartz, which showed significantly different behavior between the core and outer parts; the latter contained large amounts of $\mathrm{OH}$ and molecular water impurities, giving less radiation-induced phenomena. ${ }^{4}$

On the basis of these experimental results, a quenching mechanism of the radiation-induced phenomena for quartz samples prepared from single Madagascar rock crystal has been proposed, in which the $\mathrm{H}^{0}$-atoms resulted from a radiolysis product from $\mathrm{OH}$ and molecular water impurities can operate as a killer of radiation-induced phenomena derived from the Al-hole centers. ${ }^{4,5}$

In this paper, the quenching model concerning $\mathrm{H}^{0}$ radicals and Al-centers in electron spin resonance (ESR) spectra has

*Corresponding author. E-mail: thashi@sc.niigata-u.ac.jp. FAX $+81-25-262-6116$. been further investigated using differently originated quartz specimens.

\section{Experimental}

In the present studies, eight kinds of quartz sample differently originated from Fukushima (Japan), India, Scotland, Madagascar, Keirin (China), Ottawa, two Brazilian, and Merck (a chemical reagent) were used. Most of the quartz samples were identified to have a hydrothermal origin and possess the blue-TL (BTL) property. The samples were sieved to adjust grain sizes ranging from 150 to $250 \mu \mathrm{m}$ in a diameter for the ESR measurements.

The Al-impurity contents in the differently originated quartz samples were nondestructively determined by an instrumental neutron activation analysis (INAA) using the purest thermal neutron field as described in the previous publications. ${ }^{89}$ As mentioned previously, ${ }^{8}$ the use of the purest thermal neutrons is essential to determine accurately trace amounts of $\mathrm{Al}$ by the INAA, because the detection criterion of $\mathrm{Al}$ in the quartz is significantly dependent on the interfering reaction of ${ }^{28} \mathrm{Si}(\mathrm{n}, \mathrm{p}){ }^{28} \mathrm{Al}$ owing to the concomitant fast neutrons and matrix nuclide ${ }^{28} \mathrm{Si}$ (possessing $92.2 \%$ in abundance); even a complete pure synthetic quartz glass without $\mathrm{Al}$-impurity has been evaluated to be over 200ppm pseudo Al contents when using a normal neutron field of reactor such as in Cd-ratios of 3-4.

The quartz samples of different origins were irradiated at $77 \mathrm{~K}$ (in liquid nitrogen). The dose rate was about $15 \mathrm{kGy} / \mathrm{hr}$ at the central position of ${ }^{60} \mathrm{Co}$-source in KURRI (Kyoto University Research Reactor Institute). The total dose applied at the center position of the source was fixed to $20 \mathrm{kGy}$ for the ESR-measurements. After $\gamma$-ray irradiation, the sample was stored in $77 \mathrm{~K}$. The irradiated quartz sample was transferred from the glass vial, bearing brown color centers, into a nonirradiated quartz capillary tube for subsequent ESR measurements.

Both hydrogen radicals and Al-centers produced by $\gamma$-ray irradiation of the quartz grains were measured at $77 \mathrm{~K}$ with an ESR spectrometer (JEOL Ltd. JES-TE 200) at microwave power level of $0.03 \mu \mathrm{W}$ and $2 \mathrm{~mW}$, respectively. Under these conditions of the microwave power level, any saturation of the 
signals from all quartz samples was not observed. Results from the first ESR measurements are called hereafter an initial Al-center signal because of direct measurement after irradiation at $77 \mathrm{~K}$. Subsequently, the sample was warmed up to room temperature for a few hours and the ESR signals were measured again at $77 \mathrm{~K}$. Hereafter, this result is called second Alcenter signal.

Isochronal annealing treatments for irradiated quartz samples were performed by blowing nitrogen gas and holding at constant temperatures from $77 \mathrm{~K}$ up to the room temperature. The quartz grain sample was kept at each annealing temperature for 3 minutes and the ESR spectrometry was repeatedly conducted at $77 \mathrm{~K}$.

The BTL glow curve was measured with a conventional TLmeasuring system by operating the heating rate of $1{ }^{\circ} \mathrm{C} / \mathrm{s} .{ }^{10}$ The BTL intensities were estimated by integrating the higher temperature regions of $260-380^{\circ} \mathrm{C}$ in glow curve.

\section{Results and Discussion}

Before $\gamma$-ray irradiation, no ESR signal was observed in all natural quartz because of a simple annealing treatment for a few minutes at $400^{\circ} \mathrm{C}$. Figure 1 shows typical two ESR spectra from the $\gamma$-irradiated quartz grains. Two ESR-magnetic field regions were employed for the hydrogen radical and respective $\mathrm{Al}$-center (initial and second) measurements at $77 \mathrm{~K}$ before and after warming up to room temperature after irradiation at $77 \mathrm{~K}$. In Figure 1-(a) for an initial spectrum, two typical lines with equal intensity caused by the hydrogen radical appear in a separation of about $52 \mathrm{mT}$ due to the hyperfine splitting of nuclear magnetic spin $(I=1 / 2)$. This $\mathrm{H}^{0}$ signal in quartz was in good concordant with the previous papers. ${ }^{11,12}$ Each line has a width at half maximum amplitude of $\delta \mathrm{H} \fallingdotseq 0.01 \mathrm{mT}$ by interpolating Al-hole centers, which could not be admitted in this figure owing to delaying saturation feature in comparison with quick power saturation of $\mathrm{H}^{0}$ signal. A power saturation curve of $\mathrm{H}^{0}$ signals is illustrated in Figure 1-(c), in which square root val-

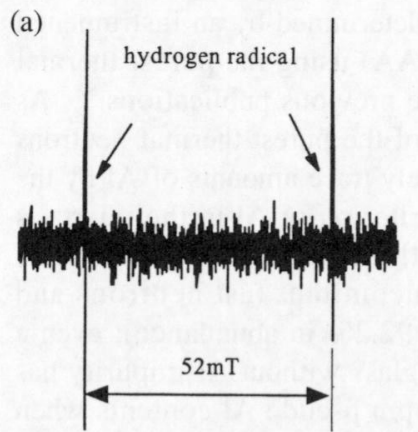

(b)

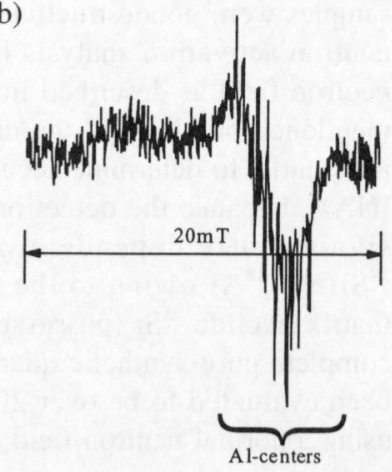

(c)

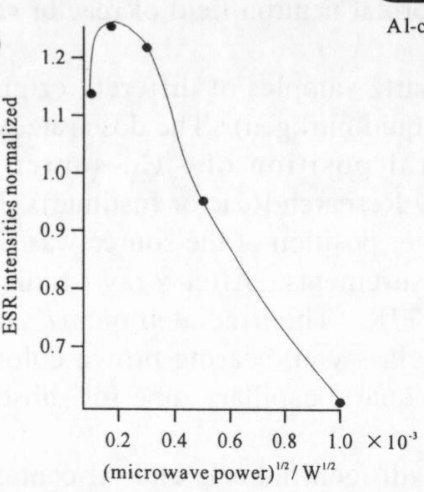

Figure 1. ESR spectra of hydrogen radicals (a) and Al-hole centers (b) and power saturation curve (c) of hydrogen radicals $\left(\mathrm{H}^{0}\right)$ from quartz grains.

Crushed Madagascar quartz grains were irradiated by $\gamma$-ray of $20 \mathrm{kGy}$ at $77 \mathrm{~K}$ and ESR measurements were carried out at $77 \mathrm{~K}$. (a) and (b) were measured with the power level of $0.03 \mathrm{~mW}$ and $2 \mathrm{~mW}$, respectively, after storage for a few hours at $77 \mathrm{~K}$. The applied power to the hydrogen radicals was determined from power saturation curve (c). ues of microwave power are plotted against $\mathrm{H}^{0}$ signal. From this result the power of $0.03 \mu \mathrm{W}$ was employed to give a maximum $\mathrm{H}^{0}$ signal.

On the other hand, Figure 1-(b) shows a well-known Alcenter signal due to $\left\{\left[\mathrm{AlO}_{4} \mathrm{~h}^{+}\right]^{0}\right\}$, which is consisting in the hyperfine (hf) structure arising from the interaction of unpaired hole-like spin with the nuclear spin $\mathrm{I}=5 / 2$ of the ${ }^{27} \mathrm{Al}$ ion. From the previous experiment, ${ }^{5}$ the applied power for Al-signal measurements was fixed to be $2 \mathrm{~mW}$ during the present measurements. Compared with different ESR-saturation behavior between $\mathrm{H}^{0}$ and $\mathrm{Al}$-center signals, the $\mathrm{H}^{0}$ atoms are considered to exist in an isolated site, presumably interstitial position, because $\mathrm{H}^{0}$-property of non-interaction with the surrounding lattices might cause more rapid power saturation than Al-center signals arising from the substitutional $\mathrm{Al}$.

Initial $\mathrm{Al}$ center intensities are plotted against the $\mathrm{Al}$-contents up to $300 \mathrm{ppm}$ as presented in Figure 2. There exist approximately linear relationships with a correlation factor of 0.89 . This result should be explained that the Al-center formation at $77 \mathrm{~K}$ occurs mainly around the substituted $\mathrm{Al}$ impurity sites, of which one bond presumably is consisting of charge compensator of $\mathrm{H}$ ions. If alkaline ions serve for the main charge compensators, the formation of Al-centers would be retarded at $77 \mathrm{~K}$ because of immobilization of alkaline ions as clarified by Hallibuton et al. ${ }^{13}$ This result means that the present natural quartz of hydrothermal origin contain prominently hydrogen charge compensator instead of alkaline ions. ${ }^{14}$ In fact, ESR-signal intensities of the hydrogen radicals showed fairly well proportional relation to that of the initial Al-centers.

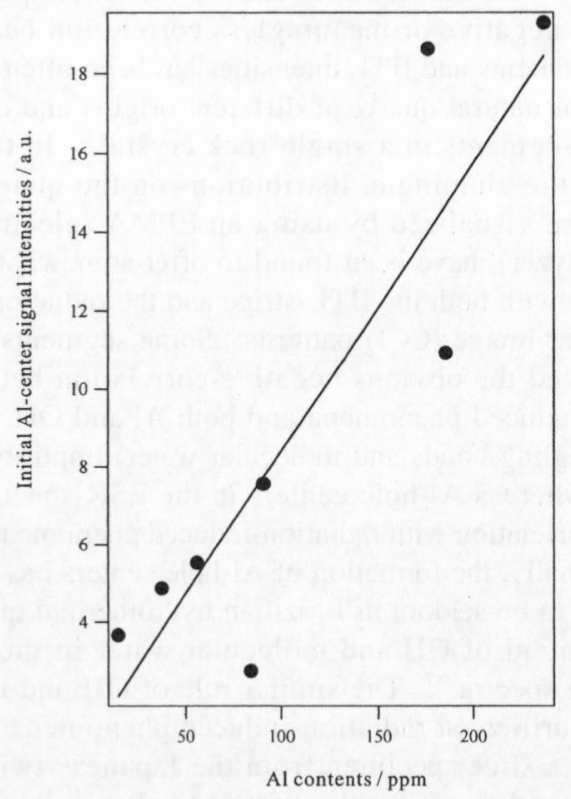

Figure 2. Dependence of initial Al-hole center signals on the Alimpurity contents.

The changes of $\mathrm{H}^{0}$ and Al-center signals are presented in Figure 3, in which Keirin and Ottawa quartz samples were selected as two examples in respect of hydrogen radical intensities. Two hydrogen peaks appear in somewhat different position estimated about $0.007 \mathrm{mT}$ regarding the applied magnetic field. Among 7 kinds of BTL quartz, maximum peak difference was $0.027 \mathrm{mT}$. This shift might be attributed to the difference of the Pauli exclusion force causing the increase of the splitting width between two hydrogen radical peaks. ${ }^{11,15}$ In the preliminary experiments, the splitting width in some feldspars has been broadened to $4 \mathrm{mT}$ in comparison with that of the quartz. These results suggest that the splitting width presumably contains unknown information related to geologically different origins of quartz.

It was noteworthy that any hydrogen radical signals produced by irradiation at $77 \mathrm{~K}$ completely disappeared in every 
(a) Hydrogen radicals

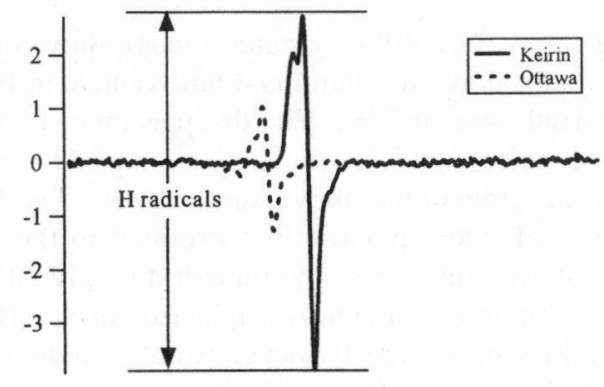

(b) Al-centers (Keirin)

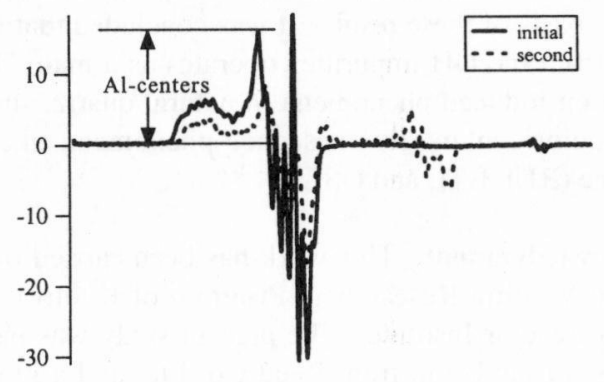

(c) Al-centers (Ottawa)

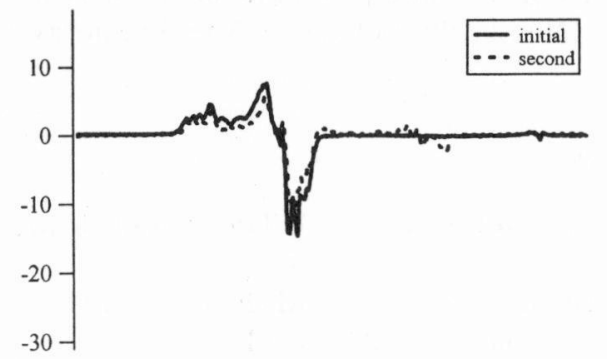

Figure 3. Two typical hydrogen signals in low magnetic field side (a) and changes of Al-hole center spectra before (b) and after (c) warming up to room temperature.

The samples were obtained from Keirin and Ottawa quartz and the ESR-measurements were carried out in the same way as described in Figure 1, except for warming treatment up to room temperature. Each signal intensity is estimated as indicated in the present figures. The hydrogen radicals from all $\gamma$-irradiated natural quartz were completely disappeared after warming procedure.

sample when the irradiated natural quartz was warmed once up to room temperature as described in our previous papers. ${ }^{5,16}$ Similar results from synthetic quartz and glasses have been already confirmed with incorporation of water component. ${ }^{17,18}$

On the other hand, the $\mathrm{Al}$ center signals maintain more or less characteristic patterns between initial spectrum without temperature elevation and second spectra after warming once up to room temperature, as seen in Figures 3-(b) and (c). Both ESR-intensities due to $\mathrm{H}^{0}$ and $\mathrm{Al}$-centers were indicated in this figure. We found meaningless correlation between hydrogen signals and initial Al-centers for natural quartz used in this study. However, when the difference between initial and second $\mathrm{Al}$ signals are taken into account by comparison with the hydrogen signals, it appears meaningful relationships from sample to sample.

With these ESR spectrum changes, the reducing intensities of Al-centers between initial and second measurements were examined as a function of the $\mathrm{H}^{0}$ intensities. The result is illustrated in Figure 4, in which there certainly exists the positive correlation between them. This result strongly supports that mobile hydrogen radicals could react easily with the initial Al-centers during the elevation of temperature to cause the corresponding elimination of Al-centers. This mechanism considered hydrogen radical as a killer of $\mathrm{Al}$-center has been proposed. ${ }^{4,5}$

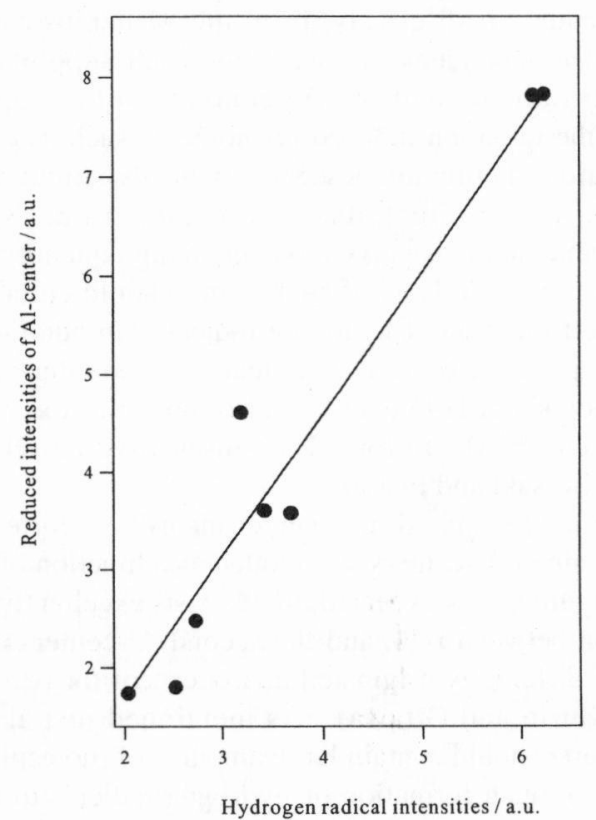

Figure 4. Correlation of the reducing intensities of Al-centers and hydrogen radicals from quartz samples of different origins. The difference between initial and second $\mathrm{Al}$-intensities after warming treatment is corresponding to the reducing intensities of Al-centers.

(a)

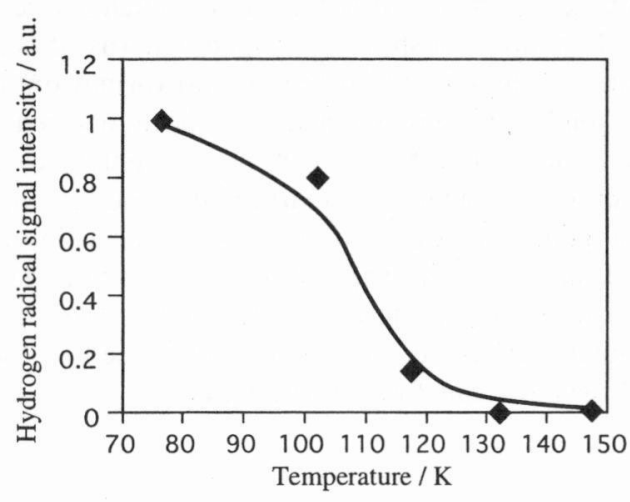

(b)

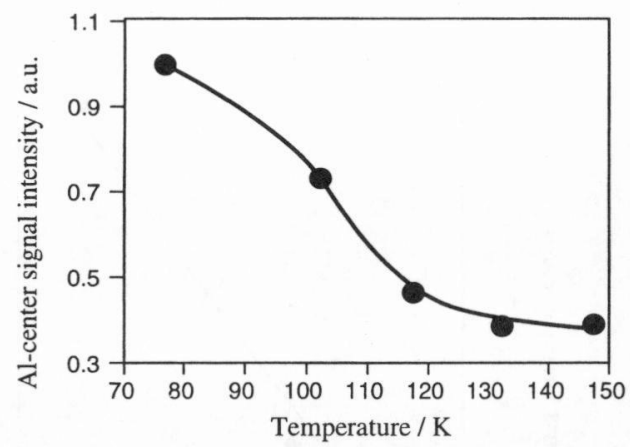

Figure 5. Isochronal annealing curves of the hydrogen radical (a) and Al-center intensities (b) from Madagascar quartz. The former signal is completely disappeared over $130 \mathrm{~K}$, whereas the Al-center signal remains beyond $140 \mathrm{~K}$.

To confirm the present elimination mechanism of Al-centers, an isochronal annealing behavior was studied between hydrogen radicals and $\mathrm{Al}$-centers. The results are indicated in Figure 5. Both curves tend to the similar decreasing trend with increments of temperature. Prominent decrease of both ESR signals occurs around $100 \mathrm{~K}$, of which temperature agrees well with the results by Week and Abraham. ${ }^{17}$

The similar behavior of both signals with annealing implies that some portions of $\mathrm{Al}$-centers were disappeared by reacting with hydrogen radical to form $\left[\mathrm{Al}^{3+}\right]---\mathrm{H}^{+}-\mathrm{OSi}$ or $\mathrm{Al}-\mathrm{OH}$, resulting in ESR insensitive sites as described in preceding publications. ${ }^{5,13}$ The activation energies were approximately evaluated as $7.5 \mathrm{~kJ} / \mathrm{mol}$ and $8.5 \mathrm{~kJ} / \mathrm{mol}$ for hydrogen radicals 
and Al-centers, respectively, from the Arrhenius analysis of temperature-dependent curves. This result supports that the hydrogen radicals and the Al-centers could cooperate to decrease the radiation-induced phenomena, such as color center formation, thermoluminescence, optically stimulated luminescence, ESR radical absorption and so on. Since the hydrothermal quartz is known to contain high quantity of water molecules, the radiolysis of such water should certainly contribute the formation of hydrogen radicals. In addition to this reduction process, some $\mathrm{OH}$ radicals as a counter part of $\mathrm{H}^{0}$ from radiolysis of $\mathrm{H}_{2} \mathrm{O}$ could be also moved to the interstitial site adjacent to $\mathrm{Al}^{3+}$ to form ESR-insensitive $\mathrm{Al}-\mathrm{OH}$ as proposed by Iwasaki and Iwasaki. ${ }^{6}$

Finally the second Al-hole center intensities, corresponding to the remained Al-centers, are plotted as a function of BTL, as seen in Figure 6. As expected, there exists excellently positive correlation between BTL and the second Al-centers even in a variety of differently originated quartz except for two kinds of quartz (Keirin and Ottawa). As mentioned just above, the Keirin quartz should contain large amounts of molecular water, followed to much formation of hydrogen radicals to react the Al-centers (cf., Figure 1-(a)). On the other hand, the Ottawa sample contains extremely small amount $-\mathrm{OH}$ and molecular water, presumably owing to the quartz formation with extremely slow cooling rate. These radiation-induced mechanisms associated with the important role of hydrogen radicals could be explained well regarding the changeable radiationinduced phenomena showing striped patterns of color center, presumably dependent on the crystal formation conditions within single crystal rock which have been reported in our laboratory. ${ }^{5}$ From these results, it is again emphasized that the $\mathrm{OH}$ and water contents in quartz play important roles on the reliable geochronological or archaeological dating using ESR methods and luminescence techniques, involving TL and optically stimulated luminescence (OSL), because the naturally accumulated $\mathrm{Al}$-centers are greatly influenced by the quantity of hydrogen radicals formed with radiation.

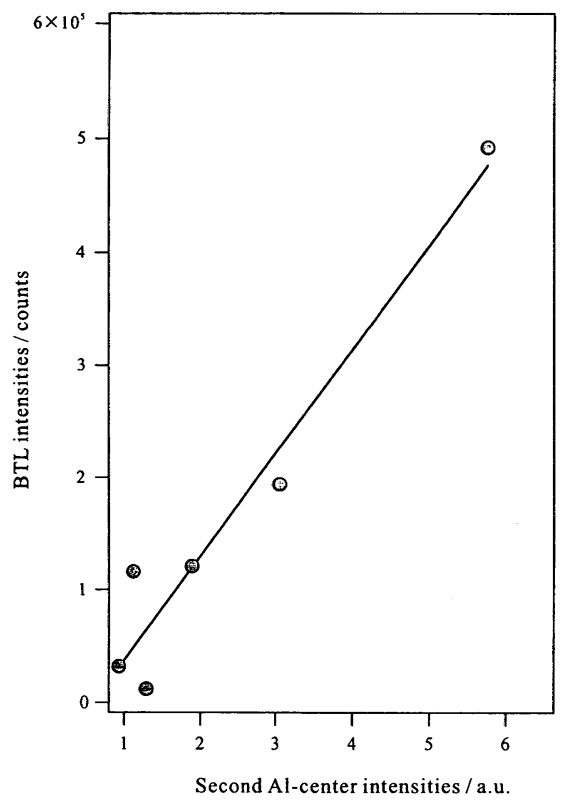

Figure 6. Dependence of BTL intensities on second Al-center signals. On the basis of the present results, both ESR and luminescence including BTL and OSL, have been proved to be available to the geochronology of white minerals, since the second Al-centers should be correlated closely with the naturally accumulated doses.

\section{Conclusions}

All of the differently originated quartz showed both the hydrogen radical and the aluminum hole centers in ESR-spectra after irradiation at $77 \mathrm{~K}$. The disappearance of hydrogen signals was coincident with the elimination of Al-centers after elevation of temperature above about $100 \mathrm{~K}$. The hydrogen radical signals were positively correlated to the reducing amounts of Al-center after the temperature elevation up to room temperature for differently originated quartz. The hydrogen radical formation greatly depends on the amounts of $\mathrm{OH}$ and molecular water in quartz, so that the quartz of different origins will offer various radiation-induced phenomena influenced by their different $\mathrm{OH}$ and water impurity contents.

On the basis of these results, it was concluded that hydrogen radicals from the $\mathrm{OH}$ impurities operates as a main "killer" of all radiation-induced phenomena in natural quartz, such as $\mathrm{Al}$ centers, color centers, luminescence phenomena like radioluminscence (RL), BTL and OSL.

Acknowledgement. This work has been carried out in part under the Visiting Researchers Program of Kyoto University Research Reactor Institute. The present study was partly supported by a foundation from Reed Co. Ltd. and a grant-in-aid for Fundamental Research from the Ministry of Education, Culture, and Sport, Japan (No. 10480024) and by the REIMEI Research (No. 2-1999) of Japan Atomic Energy Research Institute.

\section{References}

(1) S. W. S. McKeever, Nucl. Tracks Radiat. Meas. 18, 5 (1991).

(2) T. Hashimoto, S. Sakaue, M. Ichino, and H. Aoki, Nucl. Tracks Radiat. Meas. 23, 293 (1994).

(3) T. Hashimoto, S. Notoya, T. Arimura, and M. Konishi, Radiat. Meas. 26, 233 (1996).

(4) T. Hashimoto, K. Yasuda, K. Sato, H. Sakaue, and H. Katayama, Radiat. Meas. 29, 493 (1998).

(5) T. Hashimoto, H. Katayama, H. Sakaue, H. Hase, T. Arimura, and T. Ojima, Radiat. Meas. 27, 243 (1997).

(6) F. Iwasaki and H. Iwasaki, Jpn. J. Appl. Phys. 32, 893 (1993).

(7) P. L. Guzzo, F. Iwasaki, and H. Iwasaki, Phys. Chem. Minerals 24, 254 (1997).

(8) T. Hashimoto, S. Sakaue, and H. Ohira, Radioisotopes 41, 618 (1992).

(9) T. Hashimoto, T. Ojima, M. Konishi, and M. Kanemaki, Radioisotopes 44, 379 (1995).

(10)T. Hashimoto, Y. Hayashi, A. Koyanagi, K. Yokosaka, and K. Kimura, Nucl. Tracks Radiat. Meas. 11, 229 (1986).

(11) J. A. Weil, Phys. Chem. Minerals 10, 149 (1984).

(12)T. Miyazaki, N. Azuma, and K. Fueki, J. Amer. Ceram. Soc. 67, 99 (1984).

(13) L. E. Hallibuton, N. Koumvakalis, M. E. Markes, and J. J. Martin, J. Appl. Phys. 52, 3565 (1981).

(14) J. C. Newton-Howes and R. J. Fleming, Phys. Chem. Minerals 17, 27 (1990).

(15) F. J. Adrian, J. Chem. Phys. 32, 972 (1960).

(16) H. Fujita, H. Katayam, H. Hase, and T. Hashimoto, Radiat. Meas. (in press).

(17)R. A. Weeks and M. Abraham, J. Chem. Phys. 42, 68 (1965).

(18) N. Azuma, T. Miyazaki, K. Fueki, I. Sakaguchi, and S. Hirano, J. Am. Chem. Soc. 69, 19 (1960). 